\title{
Recurrent transient ischemic attacks in a moyamoya syndrome patient with ultra-long imaging follow-up
}

\author{
Lan Zhang ${ }^{1 \wedge}$, Wei Xing ${ }^{1}$, Jiajia Zhang ${ }^{2}$ \\ ${ }^{1}$ MRI Department, the First Affiliated Hospital of Henan University of Chinese Medicine, Zhengzhou, China; ${ }^{2}$ Department of Radiology, Gold \\ Coast University Hospital, Gold Coast, Australia
}

Correspondence to: Lan Zhang, MD. MRI Department, the First Affiliated Hospital of Henan University of Chinese Medicine, Zhengzhou 450000, China. Email: 13837187787@163.com; Jiajia Zhang, MD. Department of Radiology, Gold Coast University Hospital, 1 Hospital Blvd Southport, Gold Coast 4215, Australia. Email: zzjia2008@gmail.com.

Submitted Jun 16, 2021. Accepted for publication Sep 29, 2021.

doi: 10.21037/qims-21-631

View this article at: https://dx.doi.org/10.21037/qims-21-631

Moyamoya disease (MMD) is an idiopathic cerebrovascular condition characterized by progressive stenosis or occlusions of distal internal carotid arteries (ICAs) and their major branches, accompanied by the growth of a network of collateral vessels resembling a puff of smoke $(1,2)$. Moyamoya syndrome (MMS) is moyamoya-like vasculopathy that develops in patients with underlying diseases, including neurofibromatosis type 1 (NF-1), Down syndrome, Grave's disease, therapeutic irradiation, sickle cell anemia, and others (3). It has been reported that the majority of MMS patients experience symptomatic progression over a 5 -year period, which may produce transient ischemic attacks (TIAs), ischemic strokes or brain hemorrhage $(1,4,5)$. The outcome for patients is poor without appropriate treatment $(3,5)$. Due to the progressive nature, prompt diagnosis and proper management are crucial to improving the long-term prognosis of patients (6). This study reports a case involving a patient with an ultralong history of MMS. The patient received noninvasive treatment based on advanced and comprehensive imaging surveillance and, to date, has achieved good clinical outcome.

\section{Case presentation}

All procedures involving human participants were performed in accordance with the ethical standards of the institutional and/or national research committee(s) and with the Helsinki Declaration (as revised in 2013). Written informed consent was obtained from the patient for publication of this case report and the accompanying images. A copy of the written consent is available for review by the editorial office of this journal.

A 70-year-old woman was admitted to hospital due to mild paresthesia of her tongue. She had a long history of recurrent TIAs occurring over an 18-year period. In 2004, she presented initially with transient hemiplegia, but the weakness in her right limb was relieved in a few seconds. Over the past 18 years, the suspected symptoms of TIA occurred several times, with variable manifestations of partial paresthesia, amaurosis fugax, and numbness of tongue. Only the first episode was serious; subsequent attacks in the following years were comparatively mild. In the 3 years prior to her first ischemia attack, the patient's medical record showed hyperlipidemia, with no indication of hypertension or hyperglycemia. At that time, she was not taking any medicine for her hyperlipidemia. The patient was also diagnosed with Hashimoto's thyroiditis in her 30s and had been taking levothyroxine sodium for her hypothyroidism since 2001.

At the time of admission for query stroke in 2004, the patient's initial brain magnetic resonance imaging (MRI) showed only a few small foci of white matter microangiopathic changes (Figure $1 A$ ), with no acute

\footnotetext{
$\wedge$ ORCID: 0000-0002-7891-3964.
} 

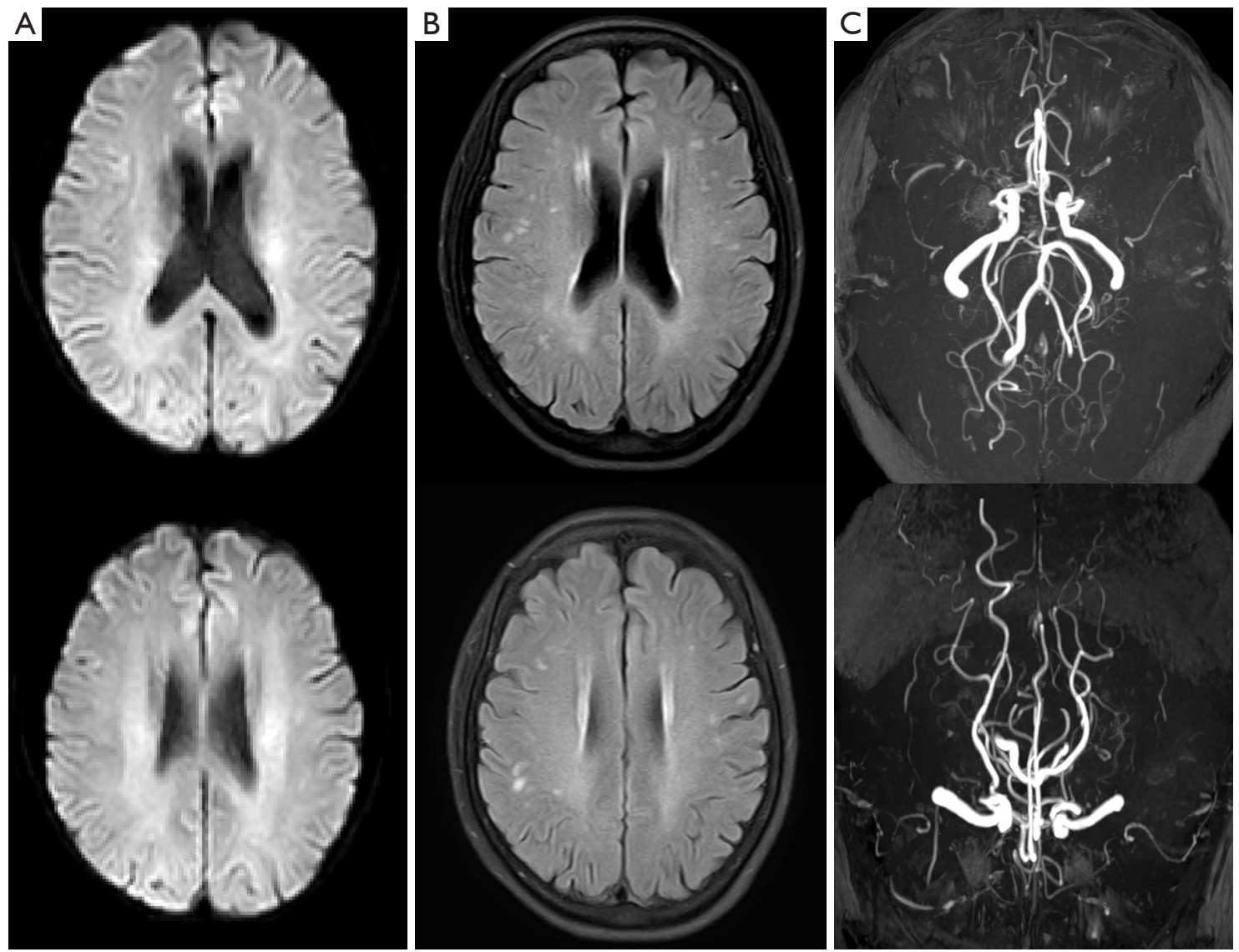

Figure 1 Initial MRI and TOF-MRA after the first onset of TIA in 2004. Only a few small foci of white matter microangiopathic changes appear on FLAIR (A), and no acute ischemic lesions are shown on DWI (B). TOF-MRA (C) demonstrates the occlusion of the bilateral MCAs and stenosis of the distal ICAs. MRI, magnetic resonance imaging; TOF-MRA, time of flight-magnetic resonance angiography; TIA, transient ischemic attacks; FLAIR, fluid-attenuated inversion recovery; DWI, diffusion-weighted imaging; MCA, middle cerebral artery; ICA, internal carotid artery.

infarction on diffusion-weighted imaging (DWI) (Figure $1 B$ ). However, incidental time of flight-magnetic resonance angiography (TOF-MRA) findings of her brain demonstrated occlusion of the bilateral middle cerebral arteries (MCAs) and stenosis of the distal ICAs, with normal anterior cerebral arteries (ACAs) and posterior cerebral arteries (PCAs) (Figure 1C). She was diagnosed with severe intracranial atherosclerosis disease, which was managed conservatively with atorvastatin and aspirin. In the following years, the patient underwent regular imaging surveillance to monitor disease progression. Her recurrent TIAs raised concern, and clinicians considered whether it was feasible for her to receive more aggressive surgery or interventional therapy. In recent years, the condition of her vessels has been reevaluated by multi-sequence MRI, as she is allergic to iodine. The steno-occlusive severity of the patient's intracranial arteries was assessed by MRA stages for MMD (7). The MRA stages were mainly determined by TOF-MRA maximum intensity projection (MIP) images, with source images used referentially. On the MRA, the steno-occlusive changes of the MCAs, ACAs and PCAs were graded as 3 stages (7): stage $1=$ no or mild stenosis $(<50 \%)$, stage $2=$ moderate stenosis $(>50 \%)$ but with intact distal segment, and stage $3=$ severe stenosis without any apparent distal segment. The patient's moyamoya-type vasculopathy could be classified as stage 3. Surprisingly, the "ivy sign" was not very prominent on fluid-attenuated inversion recovery (FLAIR) images (Figure $2 A$ ). It is well known that the "ivy sign" is the result of the slow-flowing leptomeningeal collaterals that can be detected in MMD (8). 

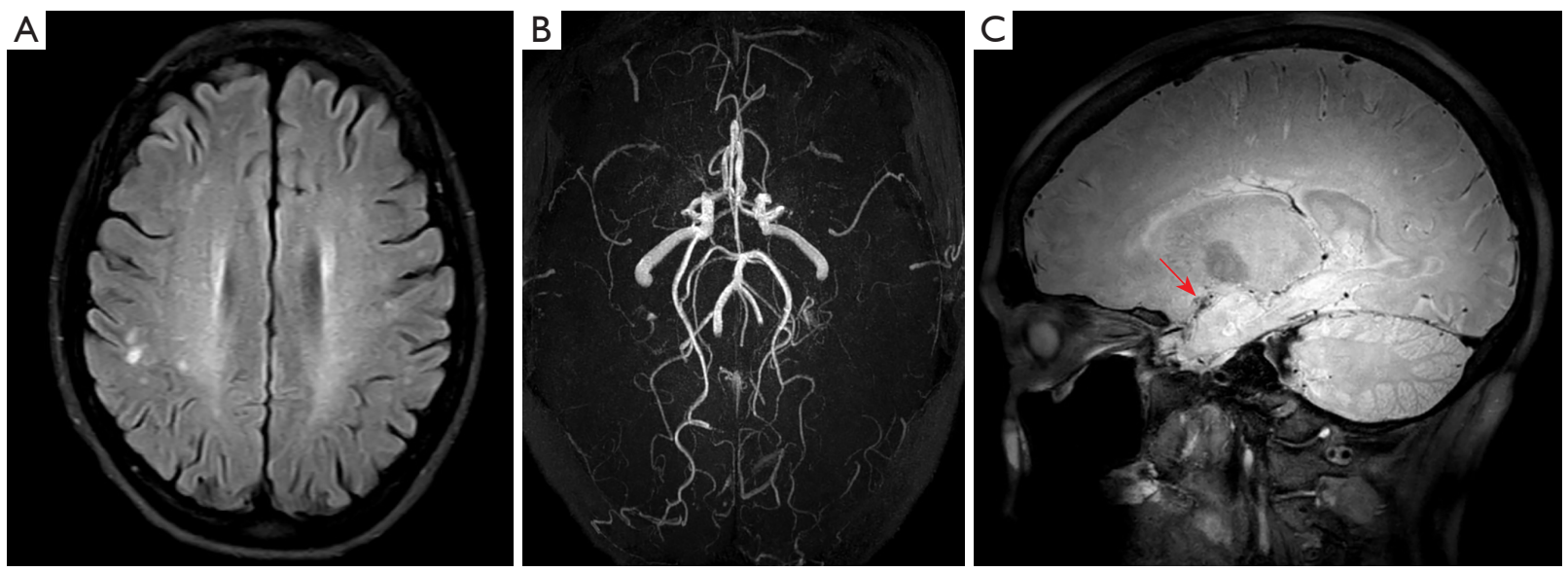

Figure 2 Brain MRI and TOF-MRA in 2013. Nonprogressive small ischemic lesions in the white matter appear on FLAIR (A). TOFMRA demonstrates the occlusion of the bilateral MCAs and collateral vessels from the bilateral PCAs and vertebrobasilar arteries (B). Highresolution vessel wall imaging shows intimal thickening and luminal occlusion of MCAs (arrow) (C). MRI, magnetic resonance imaging; TOF-MRA, time of flight-magnetic resonance angiography; FLAIR, fluid-attenuated inversion recovery; MCA, middle cerebral artery; PCA, posterior cerebral artery.

Although the characteristic "puff of smoke-like" moyamoya vessels were not apparent, her bilateral PCAs were very thick and several branches could be seen originating from the bilateral PCAs and vertebrobasilar arteries (Figure 2B), which could be interpreted as evidence of the development of collaterals from the posterior circulation. High-resolution vessel wall imaging confirmed the intimal thickening and luminal occlusion of MCAs (Figure 2C). The patient's overall imaging findings were not typical for MMD. With consideration to her medical history, the patient was eventually diagnosed with MMS by a multidisciplinary team (MDT) composed of neurologists, neurosurgeons and diagnostic and interventional radiologists.

In recent years, the patient has been monitored with frequent MRI scans under the latest noninvasive clinically available MRI techniques, such as susceptibility-weighted imaging (SWI) and arterial spin labeling (ASL). Since 2013, ASL and other MRI sequences have been carried out as routine MRI examinations on a 3.0-Tesla MRI scanner (Ingenia, Philips, Amsterdam, The Netherlands) with 16-channel head coils. Multiple brain MRIs have shown nonprogressive small white matter ischemic foci (Figure $3 A$ ), while SWI has revealed no associated microhemorrhage (Figure 3B). TOF-MRA has consistently shown the presence of collaterals from bilateral PCAs and vertebrobasilar arteries (Figure 3C).

The patient's MDT initially considered intervention or revascularization surgery as the optimal treatment to reduce the frequency of recurrent TIAs and the risk of infarction. However, repeated ASL indicated that the patient's cerebral hemodynamic impairments and collateral blood flow were not consistent with those of typical MMD. The patient's ASL data were acquired with three-dimensional pseudo-continuous ASL (3D-pCASL) using the following parameters: labeling duration $=1.5 \mathrm{~s}$, postlabeling delay $(\mathrm{PLD})=1.5 / 2.5 \mathrm{~s}$, repetition time $(\mathrm{TR})=3.7 / 4.7 \mathrm{~s}$, echo time $(\mathrm{TE})=11 \mathrm{~ms}$, field of view $(\mathrm{FOV})=240 \mathrm{~mm} \times 240 \mathrm{~mm}$ $\times 85 \mathrm{~mm}$, matrix $=64 \times 64$, thickness $=6 \mathrm{~mm}$, and duration time $=5$ minutes and 21 seconds $/ 6$ minutes and 47 seconds. In 2013, the patient's 3D-pCASL images showed serpiginous high signal intensity in the bilateral cerebral cortex and sulcus at a PLD of $1.5 \mathrm{~s}$ (Figure $4 A$ ), and, less prominently, in the bilateral temporal and parietal cortex at a PLD of 2.5 s (Figure 4B). From 2013 to 2020, 3D-pCASL at PLD of 1.5 and $2.5 \mathrm{~s}$ continuously demonstrated bright hyperintensity in the cerebral symmetric cortical surface. In 2021, the 3D-pCASL-based dynamic MRA referred to as "four-dimensional magnetic resonance angiography (4D-MRA)" and 3D-pCASL were performed on a new 3.0-Tesla MRI scanner (Ingenia CX, Philips) using 32-channel head coils. The latest ASL images showed hyperintense signal bands in the bilateral hemisphere at a PLD of $1.5 \mathrm{~s}$ (Figure $5 A$ ) and, much more prominently, at a PLD of $2.5 \mathrm{~s}$ (Figure 5B). Meanwhile, 4D-MRA data were acquired with the time-resolved angiography non-contrastenhanced (TRANCE) technique using the following 

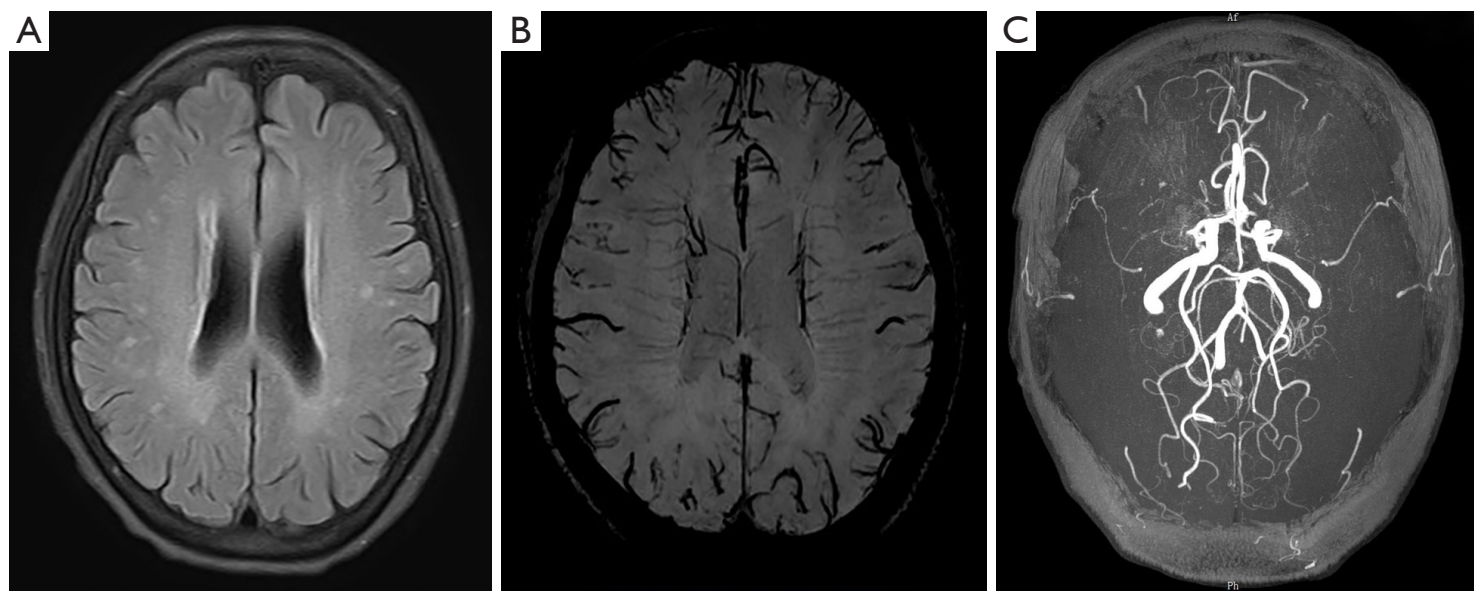

Figure 3 Brain MRI and TOF-MRA in 2021. No concerning progressive imaging findings appear on FLAIR (A), and no associated microhemorrhage appears on SWI (B). TOF-MRA shows the presence of collaterals from the bilateral PCAs and vertebrobasilar arteries (C). MRI, magnetic resonance imaging; TOF-MRA, time of flight-magnetic resonance angiography; FLAIR, fluid-attenuated inversion recovery; SWI, susceptibility-weighted imaging; PCA, posterior cerebral artery.
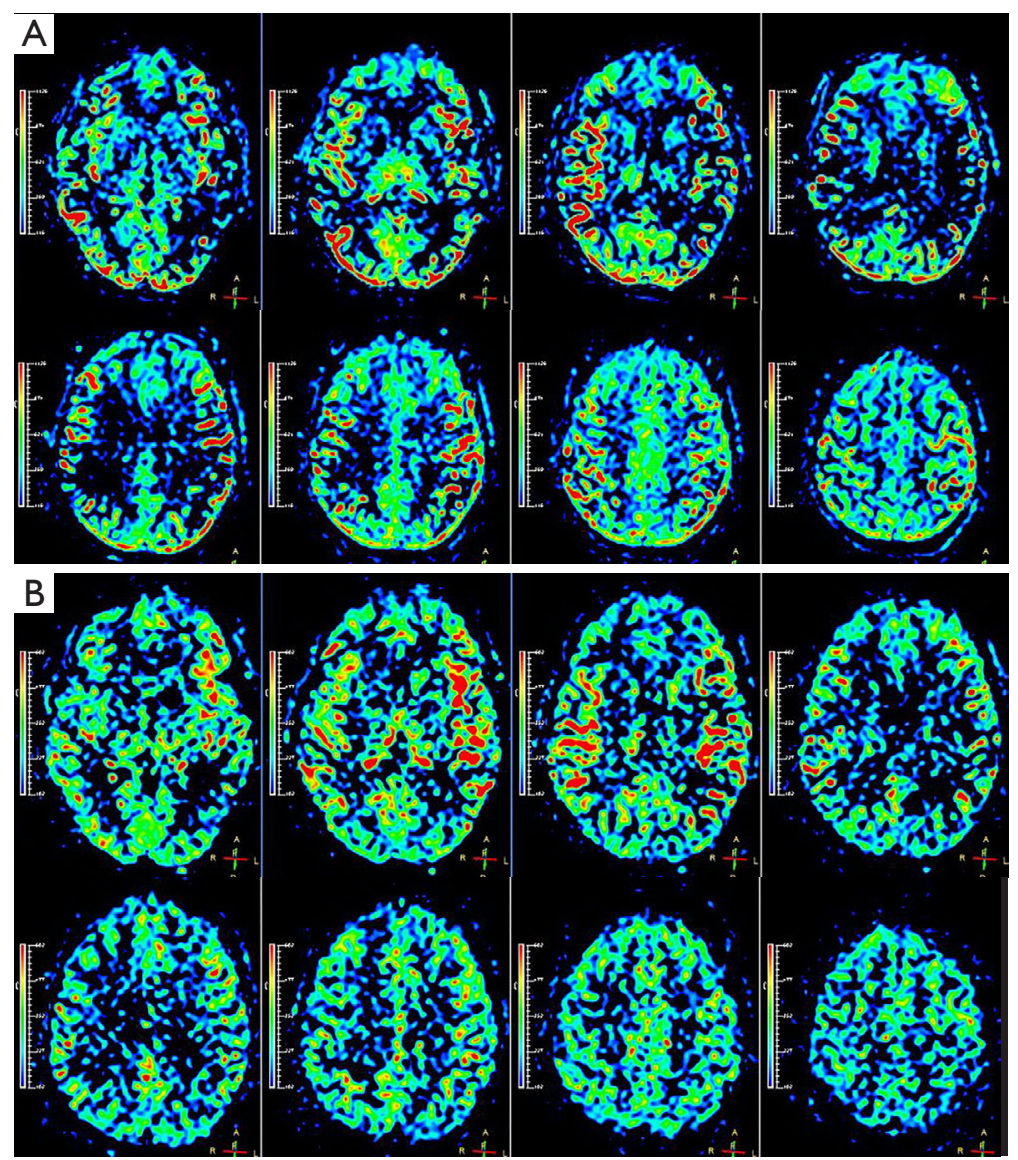

Figure 4 3D-pCASL in 2013. ASL shows serpiginous high signal intensity in the bilateral cerebral cortex and sulcus at a PLD of $1.5 \mathrm{~s}$ (A), and, less prominently, in the bilateral temporal and parietal cortex at a PLD of $2.5 \mathrm{~s}$ (B). 3D-pCASL, three-dimensional pseudo-continuous arterial spin labeling; ASL, arterial spin labeling; PLD, postlabeling delay. 

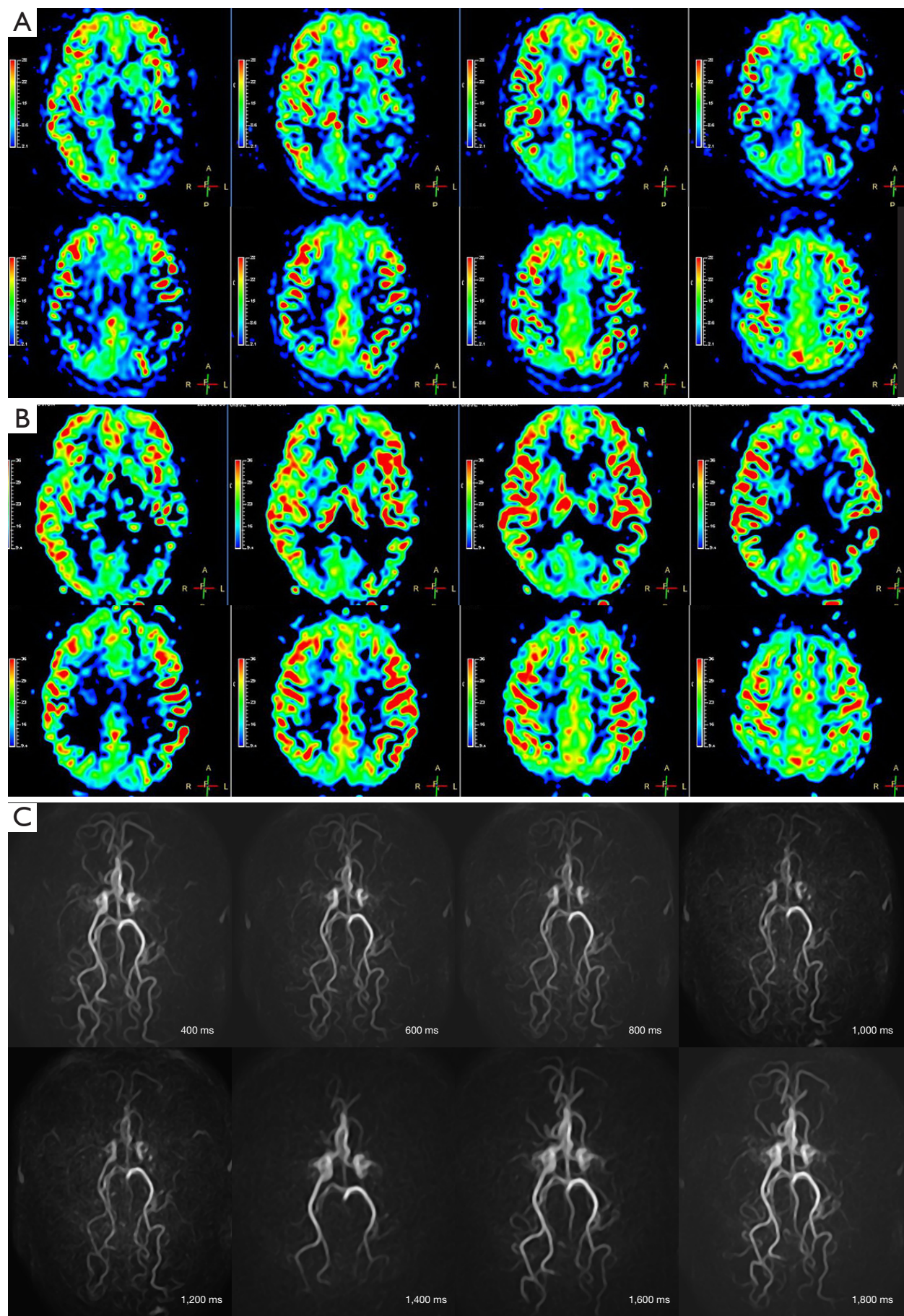

Figure 5 3D-pCASL and 4D-MRA in 2021. ASL shows hyperintense signal bands in the bilateral hemisphere at a PLD of $1.5 \mathrm{~s}$ (A) and, more prominently, in the bilateral cortex at a PLD of $2.5 \mathrm{~s}$ (B). 4D-MRA (phase interval =200 ms, phase 2 to 9) dynamically shows symmetrical emergence of the bilateral PCAs and collaterals originating from the posterior circulation (C). 3D-pCASL, three-dimensional pseudo-continuous arterial spin labeling; 4D-MRA, four-dimensional magnetic resonance angiography; ASL, arterial spin labeling; PLD, postlabeling delay; PCA, posterior cerebral artery. 
parameters: $\mathrm{TR}=9.3 \mathrm{~s}, \mathrm{TE}=4.8 \mathrm{~s}, \mathrm{FOV}=210 \mathrm{~mm} \times 210 \mathrm{~mm}$ $\times 80 \mathrm{~mm}$, spatial resolution $=1.2 \mathrm{~mm} \times 1.2 \mathrm{~mm} \times 1.3 \mathrm{~mm}$, matrix $=172 \times 130$, thickness $=1.3 \mathrm{~mm}$, label delay $=200 \mathrm{~ms}$, label $\mathrm{gap}=20 \mathrm{~mm}$, phase interval $=200 \mathrm{~ms}$, phases $=10$, and duration time $=2,200 \mathrm{~ms}$. Symmetrical emergence of bilateral PCAs was observed, and intracranial collaterals originating from the bilateral PCAs were clearly shown on the dynamic 4D-MRA (Figure 5C).

Based on MRA assessment and cerebral perfusion results, it was agreed that the patient still had sufficient cortical perfusion and the invasive therapies, such as stent implantation or revascularization surgery, posed a risk of uncontrolled hemorrhage that outweighed any potential benefit. Conservative treatment using thyroxine replacement, lipid lowering (rosuvastatin), and antiplatelet aggregation (clopidogrel) medication was then continued. The patient reported good clinical response with a much lower frequency and severity of TIAs and no progressive imaging findings.

\section{Discussion}

Moyamoya is a general term used to describe two different conditions affecting the main branches within the circle of Willis, which present distinctive findings on cerebral arteriography. These two conditions are MMD and MMS. MMS is idiopathic and more commonly seen among adults ranging in age from 20 to 40 years $(1,2)$. Studies have suggested an underlying component rather than a genetic component in MMS, which often occurs alongside autoimmune diseases, such as diabetes mellitus and thyroid disease (Grave disease or Hashimoto's thyroiditis) (3,9-11). Both conditions possibly may share a phenotype of cerebral vasculopathy, at least to some degree $(2,3)$. Once the diagnosis of MMD or MMS is made, selecting an appropriate therapeutic regimen is the next key step for the prognosis of patients.

The cause of extensive occlusion of bilateral MCAs in our patient is unknown but could be related to her hyperlipidemia and arteriosclerosis, or even to her Hashimoto's thyroiditis. Treatment options have involved daily aspirin and statins use, as well as lifestyle modifications following the first attack in 2004. Although there has been no obvious progression on surveillance imaging, the patient's TIA symptoms have not been completely relieved by medicine. Unlike typical MMD with conspicuous smoky vessels, on initial imaging, the patient showed occlusion of bilateral MCAs without regional collaterals but with prominent vessels of the posterior circulation. She was diagnosed with MMS by the MDT according to her atypical imaging findings and complex medical history. Previous reports indicate that the rate of disease progression among moyamoya patients is high, and medicine alone does not halt progression (12). Although there is no established standard regarding the optimal time for surgery, the rate of symptomatic progression is significantly reduced after surgical revascularization $(13,14)$. Given the complicated hemodynamics associated with either MMD or MMS, the assessment of cerebral perfusion state is critical for treatment planning (15). Since changes in hemodynamics are closely associated with disease progression, recurrent TIA may indicate cerebral hemodynamic impairment and imminent risk of ischemic stroke. Cerebral hemodynamic status should be carefully evaluated before and after intervention or surgery $(16,17)$.

ASL can be used as a noninvasive preoperative assessment to qualify cerebral perfusion and to monitor the alterations of hemodynamics (18-20). Moreover, ASL can help grade collateral flow and may complement digital subtraction angiography (DSA) findings $(21,22)$. As regards this patient, hypoperfusion in the bilateral MCA territories was thought to be caused by occlusion of the bilateral MCAs. Unexpectedly, there were serpiginous bright signals in the bilateral cerebral cortex and sulcus on ASL images. The bright intravascular signal known as arterial transit artifact (ATA) on ASL may indicate the presence and degree of collateral perfusion in moyamoya patients $(23,24)$. The ATAs produce hyperintense signals along the cerebral cortical sulcus, which is caused by delayed blood flow due to the stenosis or occlusion of main arteries, or collateral flow accompanying the stenosis or occlusion $(25,26)$. Investigating the cause of the ATA is necessary to evaluate the pathophysiology of patients. As the arterial transit time of collateral blood flow is longer than that of normal anterograde blood flow in moyamoya patients, the intensity of ATA conveys information about the delayed arrival of blood flow and reflects the collateral flow $(23,24)$. Studies have shown that ATAs can evaluate collateral circulation with high specificity (24).

Between 2013 and 2020, the intensity of ATA on the patient's ASL images at a PLD of $1.5 \mathrm{~s}$ was much more significant than that of $2.5 \mathrm{~s}$, which suggested that the antegrade blood flow was comparatively faster. The latest ASL in 2021 showed that the intensity of ATA at a PLD of $2.5 \mathrm{~s}$ was much more intensive than before, which may suggest that the arterial transit time of the collaterals is 
longer than the anterograde blood flow. ATA is dependent on the arterial transit time and is influenced particularly by the labeling time and PLD. The decreased and slow blood flow might induce an ATA; therefore, cortical flow in moyamoya patients may resemble this hemodynamic status. The strength of the ATA alters according to the PLD value because the delayed blood flow due to stenosis or occlusion still remains in the cortical surface $(24,27)$. Regarding this patient, ASL acquisitions with both shorter and longer PLD dramatically demonstrated conspicuous ATA in the bilateral hemispheric cortex, and this status has lasted almost 10 years to date. ATA has been reported to correlate with the development of collateral circulation and to reflect the presence of leptomeningeal collaterals on DSA (28). The balance of hemodynamics and compensated perfusion of this patient relies on slow flow from the collaterals of the posterior circulation, which prolongs the mean transit times of arterial blood flow and creates ATA on 3D-pCASL images. 4D MRA is a 3D-pCASL-based dynamic MRA with long labeling duration, which can ensure visualization of the complete arterial trees from proximal to distal vessels with adequate image quality (29). The patient's latest 4D-MRA clearly shows the presence of collaterals mainly from the bilateral PCAs. Consequently, preservation and improvement of collateral blood flow can provide protection against future strokes and reduce the frequency of ischemic symptoms while effecting a concurrent reduction in collaterals.

The patient's MDT concluded that invasive treatment is not the best management option for this patient in light of her comprehensive medical conditions, as there could be a high risk of massive intracranial hemorrhage with aggressive intervention. Persistent conservative therapy with close clinical and imaging surveillance was suggested as a personalized therapeutic strategy. The patient tolerated the conservative management well and has been achieving a good work-life balance without any clinically significant neurological deficits.

Although revascularization surgery or stent implantation is usually suggested as a viable treatment for MMD or MMS in the long term, functional imaging assessment methods coupled with a MDT approach can assist in the creation of a patient-centered management plan. Evaluation of collaterals and hemodynamic perfusion is critical for moyamoya patients. Noninvasive techniques such as ASL can predict the presence and function of collaterals, which is of great significance to the therapeutic planning and surveillance of moyamoya patients. This case of successful conservative management of MMS presents an example of such a patient-centered approach and demonstrates that active treatment does not necessarily mean aggressive treatment in this clinical mode.

\section{Acknowledgments}

The authors wish good health to all the mothers of the world (the patient is Dr. Lan Zhang's mother).

Funding: This study was funded by the National Key Research and Development Program on Chinese Medicine Modernization (2019YFC1710003), and the Henan Scientific and Technological Project (212102310732).

\section{Footnote}

Conflicts of Interest: The authors have completed the ICMJE uniform disclosure form (available at https://dx.doi. org/10.21037/qims-21-631) and have no conflicts of interest to declare.

Ethical Statement: The authors are accountable for all aspects of the work in ensuring that questions related to the accuracy or integrity of any part of the work are appropriately investigated and resolved. All procedures involving human participants were in accordance with the ethical standards of the institutional and/or national research committee(s) and with the Helsinki Declaration (as revised in 2013). Written informed consent was obtained from the patient for publication of this case report and accompanying images. A copy of the written consent is available for review by the editorial office of this journal.

Open Access Statement: This is an Open Access article distributed in accordance with the Creative Commons Attribution-NonCommercial-NoDerivs 4.0 International License (CC BY-NC-ND 4.0), which permits the noncommercial replication and distribution of the article with the strict proviso that no changes or edits are made and the original work is properly cited (including links to both the formal publication through the relevant DOI and the license). See: https://creativecommons.org/licenses/by-nc-nd/4.0/.

\section{References}

1. Scott RM, Smith ER. Moyamoya disease and moyamoya syndrome. N Engl J Med 2009;360:1226-37.

2. Smith ER. Moyamoya Biomarkers. J Korean Neurosurg 
Soc 2015;57:415-21.

3. Phi JH, Wang KC, Lee JY, Kim SK. Moyamoya Syndrome: A Window of Moyamoya Disease. J Korean Neurosurg Soc 2015;57:408-14.

4. Uchino K, Johnston SC, Becker KJ, Tirschwell DL. Moyamoya disease in Washington State and California. Neurology 2005;65:956-8.

5. Liu XJ, Zhang D, Wang S, Zhao YL, Teo M, Wang R, Cao Y, Ye X, Kang S, Zhao JZ. Clinical features and long-term outcomes of moyamoya disease: a single-center experience with 528 cases in China. J Neurosurg 2015;122:392-9.

6. Kuroda S, Houkin K. Moyamoya disease: current concepts and future perspectives. Lancet Neurol 2008;7:1056-66.

7. Lee YS, Jung KH, Roh JK. Diagnosis of moyamoya disease with transcranial Doppler sonography: correlation study with magnetic resonance angiography. J Neuroimaging 2004;14:319-23.

8. Gburek-Augustat J, Sorge I, Merkenschlager A. The "Ivy-Sign" in Moyamoya Disease-From MRI Pattern to Diagnosis. Neuropediatrics 2020;51:241-4.

9. Suzuki S, Mitsuyama T, Horiba A, Fukushima S, Hashimoto N, Kawamata T. Moyamoya disease complicated by Graves' disease and type 2 diabetes mellitus: report of two cases. Clin Neurol Neurosurg 2011;113:325-9.

10. Bower RS, Mallory GW, Nwojo M, Kudva YC, Flemming KD, Meyer FB. Moyamoya disease in a primarily white, midwestern US population: increased prevalence of autoimmune disease. Stroke 2013;44:1997-9.

11. Kim SJ, Heo KG, Shin HY, Bang OY, Kim GM, Chung CS, Kim KH, Jeon P, Kim JS, Hong SC, Lee KH. Association of thyroid autoantibodies with moyamoyatype cerebrovascular disease: a prospective study. Stroke 2010;41:173-6.

12. Kuroda S, Ishikawa T, Houkin K, Nanba R, Hokari $\mathrm{M}$, Iwasaki Y. Incidence and clinical features of disease progression in adult moyamoya disease. Stroke 2005;36:2148-53.

13. Isono M, Ishii K, Kobayashi H, Kaga A, Kamida T, Fujiki M. Effects of indirect bypass surgery for occlusive cerebrovascular diseases in adults. J Clin Neurosci 2002;9:644-7.

14. Fung LW, Thompson D, Ganesan V. Revascularisation surgery for paediatric moyamoya: a review of the literature. Childs Nerv Syst 2005;21:358-64.

15. Li J, Jin M, Sun X, Li J, Liu Y, Xi Y, Wang Q, Zhao W, Huang Y. Imaging of Moyamoya Disease and Moyamoya Syndrome: Current Status. J Comput Assist Tomogr
2019;43:257-63.

16. Fan AP, Khalighi MM, Guo J, Ishii Y, Rosenberg J, Wardak M, Park JH, Shen B, Holley D, Gandhi H, Haywood T, Singh P, Steinberg GK, Chin FT, Zaharchuk G. Identifying Hypoperfusion in Moyamoya Disease With Arterial Spin Labeling and an 15O-Water Positron Emission Tomography/Magnetic Resonance Imaging Normative Database. Stroke 2019;50:373-80.

17. Lee S, Yun TJ, Yoo RE, Yoon BW, Kang KM, Choi SH, Kim JH, Kim JE, Sohn CH, Han MH. Monitoring Cerebral Perfusion Changes after Revascularization in Patients with Moyamoya Disease by Using Arterial Spinlabeling MR Imaging. Radiology 2018;288:565-72.

18. Lehman VT, Cogswell PM, Rinaldo L, Brinjikji W, Huston J, Klaas JP, Lanzino G. Contemporary and emerging magnetic resonance imaging methods for evaluation of moyamoya disease. Neurosurg Focus 2019;47:E6.

19. Zhuang C, Poublanc J, Mcketton L, Venkatraghavan L, Sobczyk O, Duffin J, Crawley AP, Fisher JA, Wu $\mathrm{R}$, Mikulis DJ. The value of a shorter-delay arterial spin labeling protocol for detecting cerebrovascular impairment. Quant Imaging Med Surg 2021;11:608-19.

20. Qiao PG, Han C, Zuo ZW, Wang YT, Pfeuffer J, Duan L, Qian T, Li GJ. Clinical assessment of cerebral hemodynamics in Moyamoya disease via multiple inversion time arterial spin labeling and dynamic susceptibility contrast-magnetic resonance imaging: A comparative study. J Neuroradiol 2017;44:273-80.

21. Noguchi T, Kawashima M, Nishihara M, Egashira Y, Azama S, Irie H. Noninvasive method for mapping CVR in moyamoya disease using ASL-MRI. Eur J Radiol 2015;84:1137-43.

22. Lou X, Ma X, Liebeskind DS, Ma N, Tian C, Lyu J, Long $\mathrm{X}, \mathrm{Ma} \mathrm{L}$, Wang DJ. Collateral perfusion using arterial spin labeling in symptomatic versus asymptomatic middle cerebral artery stenosis. J Cereb Blood Flow Metab 2019;39:108-17.

23. Zaharchuk G, Do HM, Marks MP, Rosenberg J, Moseley ME, Steinberg GK. Arterial spin-labeling MRI can identify the presence and intensity of collateral perfusion in patients with moyamoya disease. Stroke 2011;42:2485-91.

24. Ukai R, Mikami T, Nagahama H, Wanibuchi M, Akiyama Y, Miyata K, Mikuni N. Arterial transit artifacts observed by arterial spin labeling in Moyamoya disease. J Stroke Cerebrovasc Dis 2020;29:105058.

25. Akiyama T, Morioka T, Shimogawa T, Haga S, Sayama 
T, Kanazawa Y, Murao K, Arakawa S. Arterial SpinLabeling Magnetic Resonance Perfusion Imaging with Dual Postlabeling Delay in Internal Carotid Artery Steno-occlusion: Validation with Digital Subtraction Angiography. J Stroke Cerebrovasc Dis 2016;25:2099-108.

26. Sogabe S, Satomi J, Tada Y, Kanematsu Y, Kuwayama K, Yagi K, Yoshioka S, Mizobuchi Y, Mure H, Yamaguchi I, Abe T, Yamamoto N, Kitazato KT, Kaji R, Harada M, Nagahiro S. Intra-arterial high signals on arterial spin labeling perfusion images predict the occluded internal carotid artery segment. Neuroradiology 2017;59:587-95.

27. Yoo RE, Yun TJ, Rhim JH, Yoon BW, Kang KM, Choi SH, Kim JH, Kim JE, Kang HS, Sohn CH, Han MH.

Cite this article as: Zhang L, Xing W, Zhang J. Recurrent transient ischemic attacks in a moyamoya syndrome patient with ultra-long imaging follow-up. Quant Imaging Med Surg 2022;12(3):2144-2152. doi: 10.21037/qims-21-631
Bright vessel appearance on arterial spin labeling MRI for localizing arterial occlusion in acute ischemic stroke. Stroke 2015;46:564-7.

28. Kronenburg A, Bulder MMM, Bokkers RPH, Hartkamp NS, Hendrikse J, Vonken EJ, Kappelle LJ, van der Zwan A, Klijn CJM, Braun KPJ. Cerebrovascular Reactivity Measured with ASL Perfusion MRI, Ivy Sign, and Regional Tissue Vascularization in Moyamoya. World Neurosurg 2019;125:e639-50.

29. Suzuki Y, Fujima N, van Osch MJP. Intracranial 3D and 4D MR Angiography Using Arterial Spin Labeling: Technical Considerations. Magn Reson Med Sci 2020;19:294-309. 